\title{
Characterization of a periodically driven chaotic dynamical system
}

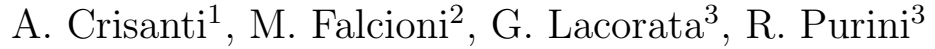 \\ and A. Vulpiani ${ }^{1}$ \\ (May, 1996) \\ ${ }^{1}$ Dipartimento di Fisica, Università "La Sapienza", I-00185 Roma, Italy \\ and Istituto Nazionale di Fisica della Materia, Sezione di Roma \\ 2 Dipartimento di Fisica, Università "La Sapienza", I-00185 Roma, Italy \\ and Istituto Nazionale di Fisica Nucleare, Sezione di Roma \\ 3 Istituto di Fisica dell' Atmosfera - CNR, P.le L. Sturzo 31 I-00144 Roma, Italy
}

\begin{abstract}
We discuss how to characterize the behavior of a chaotic dynamical system depending on a parameter that varies periodically in time. In particular, we study the predictability time, the correlations and the mean responses, by defining a local-in-time version of these quantities. In systems where the time scale related to the time periodic variation of the parameter is much larger than the "internal" time scale, one has that the local quantities strongly depend on the phase of the cycle. In this case, the standard global quantities can give misleading information.

05.45.+b,05.40.+j
\end{abstract}

Typeset using REVTEX 


\section{INTRODUCTION}

The forecast of the behavior of a system when its evolution law is known, is a problem with an obvious interest in many fields of scientific research. Roughly speaking, within this problem two main areas of investigation may be identified:

A) The definition of the "predictability time". If one knows the initial state of a system, with a precision $\delta_{o}=|\delta \boldsymbol{x}(0)|$, what is the maximum time $T_{p}$ within which one is able to know the system future state with a given tolerance $\delta_{\max } ?$

B) The understanding of the relaxation properties. What is the relation between the mean response of a system to an external perturbation and the features of its unperturbed state [1]? Using the terminology of statistical mechanics, one wants to reduce "non equilibrium" properties, such as relaxation and responses, to "equilibrium" ones, such as correlation functions [2].

A remarkable example of type- $A$ problem is the weather forecasting, where one has to estimate the maximum time for which the prediction is enough accurate. As an example of type- $B$ problem, of geophysical interest, one can mention a volcanic eruption which induces a practically instantaneous change in the temperature. In this case it is relevant to understand how the difference between the atmospheric state after the eruption and the hypothetical unperturbed state without the eruption evolves in time. In practice one wants to understand how a system absorbs, on average, the perturbation $\delta f(\tau)$ of a certain quantity $f-e . g$. the

temperature - just looking at the statistical features, as correlations, of $f$ in the unperturbed regime. This is the so called fluctuation/relaxation problem [3].

As far as problem- $A$ is concerned, in the presence of deterministic chaos, a rather common situation, the distance between two initially close trajectories diverges exponentially:

$$
|\delta \boldsymbol{x}(t)| \sim \delta_{o} \exp (\lambda t)
$$

where $\lambda$ is the maximum Lyapunov exponent of the system [4. From (11) it follows: 


$$
T_{p} \sim \frac{1}{\lambda} \ln \left(\frac{\delta_{\max }}{\delta_{o}}\right) .
$$

Since the dependence on $\delta_{\max }$ and $\delta_{o}$ is very weak, $T_{p}$ appears to be proportional to the inverse of the Lyapunov exponent. We stress however that (2) is just a naive answer to the predictability problem, since it does not take into account the following relevant features of the chaotic systems:

i) The Lyapunov exponent is a global quantity, i.e. it measures the average exponential rate of divergence of nearby trajectories. In general there are finite-time fluctuations of this rate, described by means of the so called effective Lyapunov exponent $\gamma_{t}(\tau)$. This quantity depends on both the time delay $\tau$ and the time $t$ at which the perturbation acted [5]. Therefore the predictability time $T_{p}$ fluctuates, following the $\gamma$-variations [6].

ii) In systems with many degrees of freedom one has to understand how a perturbation grows and propagates trough the different degrees of freedom [7]. In fact, one can be interested in the prediction on certain variables, e.g., those associated with large scales in weather forecasting, while the perturbations act on a different set of variables, e.g., those associated to small scales.

iii) If one is interested into non-infinitesimal perturbations, and the system possess many characteristic times, such as the eddy turn-over times in fully developed turbulence, then $T_{p}$ is determined by the detailed mechanism of propagation of the perturbations through different degrees of freedom, due to nonlinear effects. In particular, $T_{p}$ may have no relation with $\lambda[8]$.

In addition to these points, when the evolution of a system is ruled by a set of differential equations,

$$
d \boldsymbol{x} / d t=\boldsymbol{f}(\boldsymbol{x}, t)
$$

which depend periodically on time, 


$$
\boldsymbol{f}(\boldsymbol{x}, t+T)=\boldsymbol{f}(\boldsymbol{x}, t),
$$

one can have a kind of "seasonal" effect, for which the system shows an alternation, roughly periodic, of low and high predictability. This happens, for example, in the recently studied case of stochastic resonance in a chaotic deterministic system, where one observes a roughly periodic sequence of chaotic and regular evolution intervals [9].

As far as problem-B is concerned, it is possible to show that in a chaotic system with an invariant measure $P(\boldsymbol{x})$, there exists a relation between the mean response $\left\langle\delta x_{j}(\tau)\right\rangle_{P}$ after a time $\tau$ from a perturbation $\delta x_{i}(0)$, and a suitable correlation function [10]. Namely, one has the following equation:

$$
R_{i j}(\tau) \equiv \frac{\left\langle\delta x_{j}(\tau)\right\rangle_{P}}{\delta x_{i}(0)}=\left\langle x_{j}(\tau) \frac{\partial S(\boldsymbol{x}(0))}{\partial x_{i}}\right\rangle_{P},
$$

where $S(\boldsymbol{x})=-\ln P(\boldsymbol{x})$. Equation (5) ensures that the mean relaxation of the perturbed system is equal to some correlation of the unperturbed system. Of course since, in general, one does not know $P(\boldsymbol{x})$, eq. (5) provides only a qualitative information.

In this paper we discuss how one has to reformulate the predictability problem, both of type $A$ and of type $B$, for systems where the "seasonal" effects are important, i.e., when some relevant characteristic times change, in a substantial way, periodically in time.

In section \we discuss how to approach the predictability problem in systems with time periodic evolution laws. Section [II is devoted to the study of a toy model whose behavior, in spite of the simplicity of the model, catches the basic features of a system with seasonal effects. In section IV we show the results of numerical experiments, that illustrate the relevance of the concepts introduced in section [I]

\section{THE CHARACTERIZATION OF SYSTEMS WITH PERIODIC EFFECTS}

We consider systems in which one can identify two time-scales: the first, that we call $T_{E}$, can be thought as due to the coupling with an external time-dependent driver; the second, an "internal" time-scale $T_{I}$, characterizes the system in the limit of a constant external 
coupling, that we call the "stationary limit". In the following, the external time dependence will be assumed periodic with period $T$, so that $T_{E}=T$.

If the system, in the stationary limit, is chaotic, one can take as the internal time-scale the Lyapunov time, i.e., the inverse of the maximum Lyapunov exponent, $T_{I} \sim 1 / \lambda$.

In the case $T_{E} \gg T_{I}$ one can assume that the system is adiabatically driven through different dynamical regions, so that on observation times short with respect to the long external time, it evolves on a local (in time) attractor. If during its tour across the slowly changing phase space the system visits regions where the effective Lyapunov exponents are enough different, then the system shows up sensibly different predictability times, that may occur regularly when the driver is time-periodic. Consider, for instance, a slight modification of the Lorenz model [11], which is the first geophysical dynamical system where deterministic chaos has been observed:

$$
\left\{\begin{array}{l}
d x / d t=10(y-x) \\
d y / d t=-x z+r(t) x-y \\
d z / d t=x y-\frac{8}{3} z
\end{array}\right.
$$

where the control parameter has a periodic time variation:

$$
r(t)=r_{o}-A \cos (2 \pi t / T) .
$$

Since this model describes the convection of a fluid heated from below between two layers whose temperature difference is proportional to the Rayleigh number $r$, the periodic variations of $r$ roughly mimic the seasonal changing on the solar heat inputs. When $r_{o}$ is close to the threshold $r_{c r}=24.74$, where in the standard Lorenz model a transition takes place from stable fixed points to a chaotic attractor, and the amplitude $A$ of the periodic forcing is such that $r(t)$ oscillates below and above $r_{c r}$, a good approximation of the solution for very large $T$, may be given by

$$
x(t)=y(t)= \pm \sqrt{\frac{8}{3}(r(t)-1)} \quad z(t)=r(t)-1,
$$

which is obtained from the fixed points of the standard Lorenz model by replacing $r$ with $r(t)$. The stability of this solution is a rather complicated issue, which depends on the values 
of $r_{o}, A$, and $T$. It is natural to expect that if $r_{o}$ is larger than $r_{c r}$ the solution is unstable. In this case, for $A$ large enough (at least $r_{o}-A<r_{c r}$ ) one has a mechanism similar to stochastic resonance in bistable systems with random forcing [9]. The value of $T$ is crucial: for large $T$ the systems behaves as follows. If

$$
T_{n} \simeq n T / 2-T / 4 \quad(n=1,2, \ldots)
$$

are the times at which $r(t)=r_{c r}$, one can naively expect that for $0<t<T_{1}-$ when $r(t)$ is smaller than $r_{c r}$ - the system is stable and the trajectory is close to one of the two solutions (8), while for $T_{1}<t<T_{2}$ - when $r(t)>r_{c r}$ - both solutions (8) are unstable and the trajectory relaxes toward a sort of "adiabatic" chaotic attractor. The chaotic attractor smoothly changes at varying of $r$ above the threshold $r_{c r}$, but if $T$ is large enough this dependence in first approximation can be neglected. When $r(t)$ becomes again smaller than $r_{c r}$, the "adiabatic" attractor disappears and, in general, the system is far from the stable solutions (8); but it relaxes toward them, being attractive. If the half-period is much larger than the relaxation time, in general, the system follows one of the two regular solutions (8) for $T_{2 n+1}<t<T_{2 n+2}$. However, there is a small but non-zero probability that the system has no enough time to relax to (8) and its evolution remains chaotic. Figure 11 shows the time evolution of the variable $z$, for $r_{o}=25.5$ and $A=4$, in the cases $T=300$ (a) and $T=1600$ (b). They provide an unambiguous numerical evidence that the jumps from the chaotic to the regular behavior (and the contrary) are well synchronized with $r(t)$, with probability close to 1 when the forcing period $T$ is very long (see fig. $1 \mathrm{~b}$ ). On the other hand, for small value of $T$ the system often does not perform the transition from the chaotic to the regular behavior, as seen in fig. 11 a.

It is worth stressing that the system is chaotic. In both cases, in fact the first Lyapunov exponent is positive.

It is rather clear from this example that the Lyapunov exponent is not able to characterize the above behavior, since it just refers to a very long time property of the system, i.e., a property involving times longer than $T$. A more useful and detailed information can be 
obtained by computing a "local" average of the exponential rate of divergence for initially close trajectories. By this we mean an average which explicitly depends on the time $t_{o}$, modulus the external period $T$, to test the behavior of the system in the different states of the external driver. In this way one can make evident different behaviors, if any, of the system.

We therefore define the mean effective Lyapunov exponent, for the time $t_{o} \in[0, T]$ and for a delay $\tau$, as

$$
\langle\gamma(\tau)\rangle_{t_{o}}=\lim _{N \rightarrow \infty} \frac{1}{N} \sum_{k=0}^{N-1} \gamma\left(t_{o}+k T, \tau\right)
$$

where

$$
\gamma(t, \tau)=\frac{1}{\tau} \ln \frac{|\delta \boldsymbol{x}(t+\tau)|}{|\delta \boldsymbol{x}(t)|}
$$

is the local expansion rate, and $\delta \mathbf{x}(t)$ evolves according to the linear equation

$$
\frac{d}{d t} \delta x_{i}(t)=\sum_{j} \frac{\partial f_{i}(\boldsymbol{x}(t), t)}{\partial x_{j}} \delta x_{j} .
$$

From this definition it is clear that $\langle\gamma(\tau)\rangle_{t_{o}}$ measures the growth of the distance between two trajectories that differ by $|\delta \boldsymbol{x}(t)|$ when the external driver passes through a fixed value (or state). The maximum Lyapunov exponent of the system gives the global average of $\langle\gamma(\tau)\rangle_{t_{o}}$ :

$$
\lambda=\lim _{\tau \rightarrow \infty}\langle\gamma(\tau)\rangle_{t_{o}}=\frac{1}{T} \int_{0}^{T}\langle\gamma(\tau)\rangle_{t_{o}} d t_{o}
$$

If one is interested on predictability for times much smaller then $T,\langle\gamma(\tau)\rangle_{t_{o}}$ is a more appropriate quantity than $\lambda$, since it distinguishes among different regimes. For example, in the system (6) discussed above, for the given values of the parameters, one has $\lambda>0$, but $\langle\gamma(\tau)\rangle_{t_{o}}<0$ when $t_{o} \in[(n-1 / 4) T,(n+1 / 4) T]$. In the case of weather forecasting, different values of $\gamma$ for different $t_{o}$, correspond to different degree of predictability during the year.

As far as the response properties are concerned, we expect that in a chaotic system the hypothesis of existence of "adiabatic" attractors implies that a fluctuation/relaxation relation holds also as a time-local property, provided one uses correlation and response 
functions computed according a local, not a global, average. So besides the usual correlation function between the variables $x_{i}$ and $x_{j}$,

$$
C_{i j}^{(G)}(\tau)=\overline{x_{i}(t) x_{j}(t+\tau)}-\overline{x_{i}} \overline{x_{j}}
$$

where $\overline{(\cdot)}$ indicates the global average,

$$
\overline{A_{i}}=\lim _{t \rightarrow \infty} \frac{1}{t} \int_{o}^{t} A_{i}\left(t^{\prime}\right) d t^{\prime}
$$

we introduce their correlation on a delay $\tau$ after the time $t_{o} \in[0, T]$ :

$$
C_{i j}\left(t_{o}, \tau\right)=\left\langle x_{i}\left(t_{o}\right) x_{j}\left(t_{o}+\tau\right)\right\rangle_{t_{o}}-\left\langle x_{i}\right\rangle_{t_{o}}\left\langle x_{j}\right\rangle_{t_{o}}
$$

where the local average is defined as in (10)

$$
\langle A\rangle_{t_{o}}=\lim _{N \rightarrow \infty} \frac{1}{N} \sum_{k=0}^{N-1} A\left(t_{o}+k T\right) .
$$

In a similar way, one can consider two different kinds of mean response function of the variable $x_{i}$ to a small perturbation $\delta x_{j}$ : the global average response,

$$
R_{i j}^{(G)}(\tau)=\frac{\overline{\delta x_{i}(t+\tau)}}{\delta x_{j}(t)},
$$

and the local average response for the time $t_{o}$,

$$
R_{i j}\left(t_{o}, \tau\right)=\frac{\left\langle\delta x_{i}\left(t_{o}+\tau\right)\right\rangle_{t_{o}}}{\delta x_{j}\left(t_{o}\right)}
$$

The quantity (18) gives the mean response, after a delay $\tau$, to a perturbation occurred at the time $t$, chosen at random, i.e., with uniform distribution in $[0, T]$. We shall see that $R_{i j}^{(G)}(\tau)$ can be rather different, even at a qualitative level, from $R_{i j}\left(t_{o}, \tau\right)$.

\section{A TOY MODEL}

The ideas discussed in Sec. II are illustrated here by means of a simple model. Consider the Langevin equation [12] 


$$
\frac{d}{d t} q(t)=-a(t) q(t)+\xi(t)
$$

where $\xi(t)$ is $\delta$-correlated white noise, i.e., $\xi(t)$ is a gaussian variable with

$$
\langle\xi(t)\rangle=0, \quad\left\langle\xi(t) \xi\left(t^{\prime}\right)\right\rangle=2 \Gamma \delta\left(t-t^{\prime}\right)
$$

and the coefficient $a(t)$ is a periodic function of period $T: a(t+T)=a(t)$. We require that

$$
\int_{0}^{T} d t a(t)>0
$$

to ensure a well defined asymptotic probability distribution for the stochastic process given by eq. (20). Moreover, we assume a slow variation of $a(t)$, i.e.

$$
\min _{t} a(t) \gg \frac{1}{T}
$$

so that, by making an adiabatic approximation, a "local" probability distribution exists at any time.

Without the noise term, the process described by eq. (20) is nonchaotic. Therefore the model (20) cannot exhibit the full rich behaviour of chaotic systems, nevertheless it catches some of the relevant features. It is easy to see that the characteristic decay time of the local correlation

$$
\begin{aligned}
C\left(t_{o}, \tau\right) & =\left\langle q\left(t_{o}\right) q\left(t_{o}+\tau\right)\right\rangle_{t_{o}} \\
& =\lim _{N \rightarrow \infty} \frac{1}{N} \sum_{k=0}^{N-1} q\left(k T+t_{o}+\tau\right) q\left(k T+t_{o}\right)
\end{aligned}
$$

depends on $t_{o}$. This can be easily computed by using the formal solution of (20)

$$
q(t)=G(t)\left[q(0)+\int_{0}^{t} d \tau G^{-1}(\tau) \xi(\tau)\right]
$$

where

$$
G(t)=\exp \left[-\int_{0}^{t} d \tau a(\tau)\right]
$$

A straightforward calculation leads to 


$$
C\left(t_{o}, \tau\right)=C\left(t_{o}, 0\right) G\left(t_{o}, \tau\right) / G\left(t_{o}\right)
$$

where the equal-time correlation is

$$
C\left(t_{o}, 0\right)=G^{2}\left(t_{o}\right)\left[\lim _{N \rightarrow \infty} \frac{1}{N} \sum_{k=0}^{N-1} q(k T)^{2}+2 \Gamma \int_{0}^{t_{o}} d \tau G^{-2}(\tau)\right]
$$

In fig. 22 we show $C\left(t_{o}, \tau\right) / C\left(t_{o}, 0\right)$ as a function of $\tau$ for

$$
a(t)=a+b \cos \left(\frac{2 \pi}{T} t\right), \quad a+b>0
$$

for two different values of $t_{o}$, namely $t_{o}=0$ and $t_{o}=T / 2$, with $T=10, a=1, b=-0.9$ and $\Gamma=0.5$. The different behaviour is evident. By defining a characteristic time as the time $s$ it takes to have $C\left(t_{o}, s\right)=0.1$, we get for this case $s_{0} \approx 3.475$ and $s_{T / 2} \approx 1.275$. When starting from $t_{o}=T / 2$ the decay is almost a factor 3 faster than starting from $t_{o}=0$. The usual global average,

$$
C^{(G)}(\tau)=\lim _{t \rightarrow \infty} \frac{1}{t} \int_{0}^{t} d t^{\prime} q\left(t^{\prime}\right) q\left(t^{\prime}+\tau\right)
$$

gives an average correlation function, so its characteristic decay time is not able to distinguish different regimes. Moreover, while $C\left(t_{o}, \tau\right) / C\left(t_{o}, 0\right)$ does not depend on the noise strength $\Gamma, C^{(G)}(\tau) / C^{(G)}(0)$ does. In fig. 目 we used $\Gamma=0.5$.

We consider now how the system responds at time $t_{o}+\tau$ to a perturbation done at time $t_{o}$. This is described by the mean response function $R\left(t_{o}, \tau\right)$, which can be computed as follows. One takes two trajectories differing at time $t_{o}$ by a quantity $\epsilon$, i.e., $\delta q\left(k T+t_{o}\right)=\epsilon$ for any $k$, and evolving with the same realization of noise. Then the local response function is

$$
\begin{aligned}
R\left(t_{o}, \tau\right) & =\lim _{N \rightarrow \infty} \frac{1}{N} \sum_{k=0}^{N-1} \frac{\delta q\left(k T+t_{o}+\tau\right)}{\delta q\left(k T+t_{o}\right)} \\
& =\lim _{N \rightarrow \infty} \frac{1}{N} \sum_{k=0}^{N-1} \frac{\delta q\left(k T+t_{o}+\tau\right)}{\epsilon}
\end{aligned}
$$

where $\delta q\left(k T+t_{o}+\tau\right)$ is the difference between the two trajectories at time $t_{o}+\tau$. Both times $t_{o}$ and $\tau$ run over a cycle, i.e., in the interval $[0, T]$. 
By making use of (25) it is easy to see that

$$
R\left(t_{o}, \tau\right)=\frac{G\left(t_{o}, \tau\right)}{G\left(t_{o}\right)}
$$

By combining eq. (27) and (32) we have the fluctuations/relaxation relation [2]

$$
C\left(t_{o}, \tau\right)=C\left(t_{o}, 0\right) R\left(t_{o}, \tau\right)
$$

The scenario just described remains basically valid for non-linear Langevin equation. Consider for example the equation

$$
\frac{d}{d t} q(t)=-a(t) q^{3}(t)+\xi(t)
$$

where $a(t)$ is still given by (29).

It is natural to expect that, because of the adiabatic assumption, for eq. (34) a probability distribution

$$
P_{t}(q) \propto \exp -\left[S_{t}(q)\right]
$$

with $S_{t}(q)=q^{4} a(t) / 4 \Gamma$, exists at any time. Therefore a natural ansatz is to assume that (5) becomes:

$$
R\left(t_{o}, \tau\right)=\left\langle q\left(t_{o}, \tau\right) \frac{\partial S_{t_{o}}(q)}{\partial q\left(t_{o}\right)}\right\rangle_{t_{o}}
$$

In Fig. 3 we show $R\left(t_{o}, \tau\right)$ and $\left\langle q\left(t_{o}, \tau\right)\left[\partial S_{t_{o}}(q) / \partial q\left(t_{o}\right)\right]\right\rangle_{t_{o}}$ versus $\tau$, for different $t_{o}$, with $a=1, b=-0.5, T=10$ and $\Gamma=0.5$. The two curves refer to $t_{o}=0$ and $t_{o}=T / 2$. We see that eq. (36) is well obeyed and that the characteristic decay times are different.

\section{NUMERICAL SIMULATIONS}

In this section we report the results obtained for the Lorenz model (6) with the Rayleigh parameter $r$ varying periodically in time according to eq. (7). We remind that at the value $r=r_{c}=166.07$ the standard Lorenz model has a transition from a regular evolution (stable orbit) to a regime of intermittent chaos, and the maximum Lyapunov exponent depends on 
$r>r_{c}$ through a scaling law $\lambda \sim \sqrt{r-r_{c}}$, for $r-r_{c} \ll 1$ [13]. Since we are interested in chaotic systems with a non negligible variation of the degree of chaoticity, we chose $r_{o}$ close to $r_{c}$. Let us recall that $\lambda$ changes very slowly for $r$ near $r_{c r}=24.74$, so that a periodic variation of $r$ in this region is not very interesting, at least if $r(t)>r_{c}$ for any $t$. We take $r_{o}=166.6, A=0.5$ and we consider three different periods: $T=1000,100,10$. If $T$ is very large with respect to the period $(\sim O(1))$ of the unstable periodic orbit, the variations of $r$ can be taken as quasi-adiabatic. Therefore we expect that for large $T$ and for $\tau \ll T$, the local effective Lyapunov exponent of the system varies periodically in time with the same period of r,i.e.,

$$
\langle\gamma\rangle_{t_{o}} \approx \lambda\left[r\left(t_{o}\right)\right] \sim \sqrt{r\left(t_{o}\right)-r_{c}} \propto \sqrt{\cos \left(2 \pi t_{o} / T\right)}
$$

From fig. 1 a one sees that this approximation holds true if $r\left(t_{o}\right)$ is not too far from $r_{c}$. The value of the maximum Lyapunov exponent should be close to the maximum Lyapunov exponent of the stationary Lorenz model computed at $r_{o}$, i.e., the average value of $r$ on the whole period.

When $T$ is not long enough, so that one cannot speak anymore of adiabatic attractors, the behavior of $\langle\gamma\rangle_{t_{o}}$ has no relation with that of $r(t)$, as shown in fig. $4 \mathrm{~b}$.

We discuss now the behaviour of the "local" correlation functions, defined by eq. (16), and of the "local" response functions, eq. (19). From fig. 5 one sees rather clearly how the "local" correlation functions depend rather strongly from the initial time $t_{0}$. In particular, the dependence on $t_{0}$ of the mean decay time is well evident.

The correlation functions show a rapid oscillating behaviour on times $\sim O(1)$, with a typical period of the order of the mean circulation time near the "ghost" of the stable orbit existing at $r=r_{c}$, while they decay for long delays, with characteristic times that sensibly depend on the degree of chaos relative to the initial instant.

Let us stress that, because of the highly non gaussian nature of the system, there is not a simple direct proportionality between the response $R_{i j}$ and the standard correlation $C_{i j}$, both in the global and in the local version. This happens also in autonomous systems 
10] and is not a pathological behavior. From very general arguments [10] one can show that mean response and correlation function have the same qualitative behavior, e.g., the same decaying properties on large time delay. However, the agreement between $R_{i j}$ and $C_{i j}$ is very poor for moderate delay. This is so because in chaotic systems the error bars, in the numerical computation of the mean response function, increase exponentially with the delay and, when $\tau$ is not very large, the mean response has to be compared with a suitable correlation function - see eq. (5) - which depends on the unknown invariant probability distribution $P(\boldsymbol{x})$.

\section{CONCLUSIONS}

We have discussed how to characterize the behaviour of a chaotic dynamical system when a "seasonal" effect is present, i.e. when there exist two well separated time scales: the internal one and that one of the periodic variation of a control parameter. A proper characterization has been obtained by the introduction of a restricted average for the relevant quantities: correlation functions, response functions and Lyapunov exponents. These selective averages keep into account, in an explicit way, the phase of the external period at which the system is observed. We stress that, in the presence of a "seasonal" effect, the usual global averaged quantities, can give only a rough information.

\section{ACKNOWLEDGMENTS}

MF and AV acknowledge the financial support of the Istituto Nazionale di Fisica Nucleare, through the Iniziativa specifica FI11. AC and AV acknowledge the financial support of the Istituto Nazionale di Fisica della Materia. 


\section{REFERENCES}

[1] Leith C A 1973 J. Appl. Meteor. 121066

Leith C A 1978 Nature 276352

[2] Kubo R, Toda M and Hashitsume N 1985 Statistical Physics (Berlin: Springer-Verlag)

[3] Kraichnan R H 1959 Phys. Rev. 1191181

Leith C A 1975 J. Atmos. Sci. 322022

Bell T L 1980 J. Atmos. Sci. 371700

[4] Benettin G, Galgani L and Strelcyn J M 1976 Phys. Rev. A14 2338

[5] Paladin G and Vulpiani A 1987 Phys. Rep. 156147

[6] Crisanti A, Jensen M H, Paladin G and Vulpiani A 1993 Phys. Rev. Lett. 70166

Crisanti A, Jensen M H, Paladin G and Vulpiani A 1993 J. Phys. A26 6943

[7] Pikosky A 1993 Chaos 3225

Paladin G and Vulpiani A 1994 J. Phys. A27 4911

[8] Aurell E, Boffetta G, Crisanti A, Paladin G and Vulpiani A 1996 Phys. Rev. E53 2337

Boffetta G, Paladin G and Vulpiani A 1996 to appear on J. Phys. A29

[9] Crisanti A, Falcioni M, Paladin G and Vulpiani A 1994 J. Phys. A27 L597

[10] Carnevale G F, Falcioni M, Isola S, Purini R and Vulpiani A 1991 J. Fluids A3 2247

Falcioni M, Isola S and Vulpiani A 1990 Phys. Lett. A144 341

[11] Lorenz E N 1963 J. Atmos. Sci. 20130

[12] Wax N (Editor) 1954 Noise and stochastic processes New York: Dover

[13] Manneville P and Pomeau Y 1980 Physica D1 219 


\section{FIGURES}

FIG. 1. Model (6),(河) with $r_{o}=25.5$ and $A=4 . z$ as a function of $t / T$ for (a) $T=300$ and (b) $T=1600$.

FIG. 2. Model (20), (29) with $a=1, b=-0.9, T=10$. and $\Gamma=0.5 . C^{(G)}(\tau)$ (diamonds) and $C\left(t_{o}, \tau\right) / C\left(t_{o}, 0\right)$ as functions of $\tau$ for $t_{o}=0$ (full line) and $t_{o}=T / 2=5$ (dashed line).

FIG. 3. Model (34), (29) with $a=1, b=-0.9, T=10$. and $\Gamma=0.5 . R\left(t_{o}, \tau\right)$ (full line) and $\left\langle q\left(t_{o}, \tau\right)\left[\partial S_{t_{o}}(q) / \partial q\left(t_{o}\right)\right]\right\rangle_{t_{o}}$ (full squares) as functions of $\tau$, for $t_{o}=0$ and $t_{o}=T / 2=5$.

FIG. 4. Model (6),(阮) with $r_{o}=166,6$ and $A=0,5 .\langle\gamma\rangle_{t_{o}}$ as a function of $t_{o}$ for (a) $T=1000$ and (b) $T=10$.

FIG. 5. Model (6),(7) with $r_{o}=166,6, A=0,5$ and $T=1000 . C_{33}\left(t_{o}, \tau\right)$ as a function of $\tau$ for (a) $t_{o}=0$ and (b) $t_{o}=T / 2=500$. 


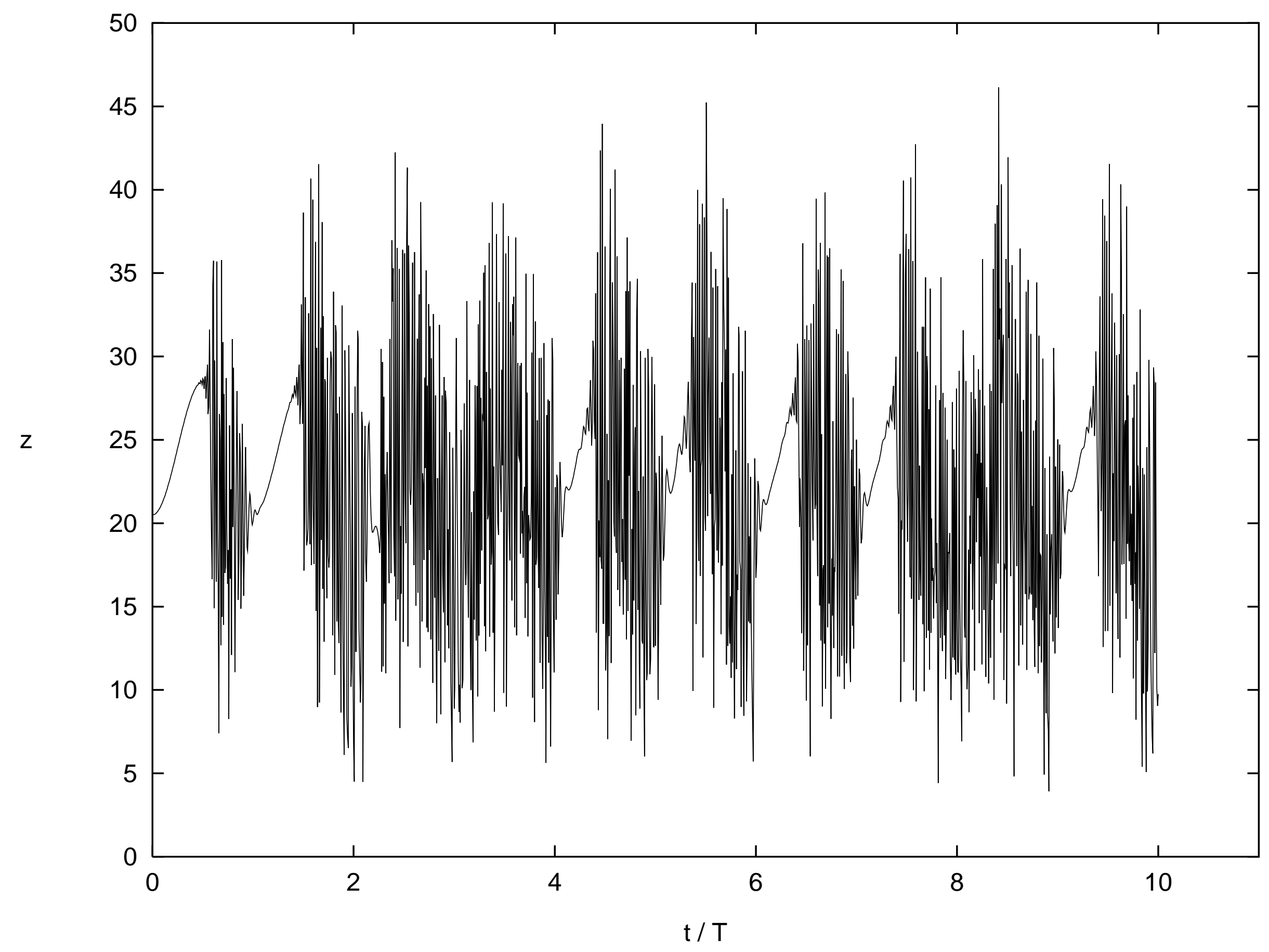









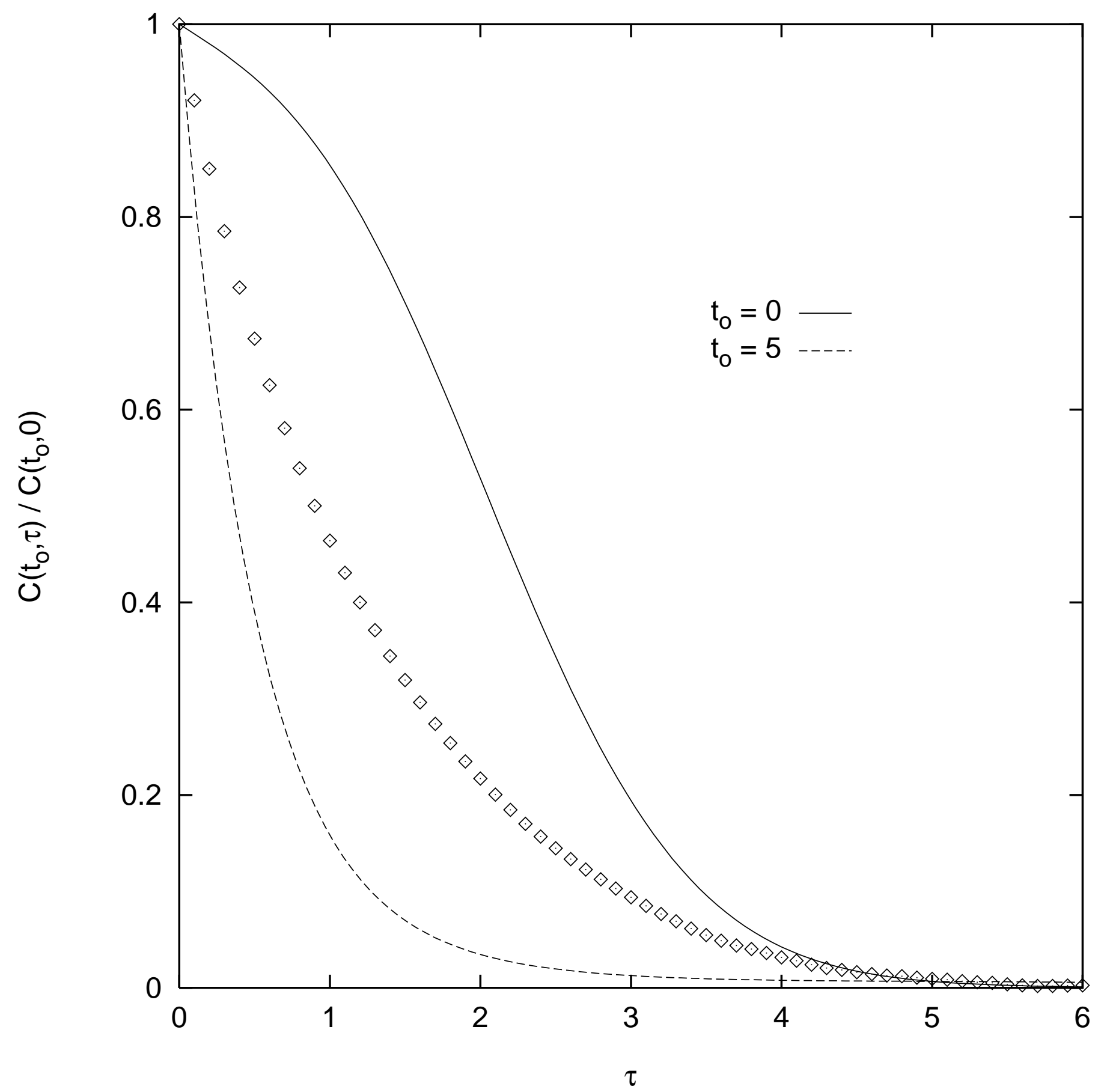

Fig. 2 


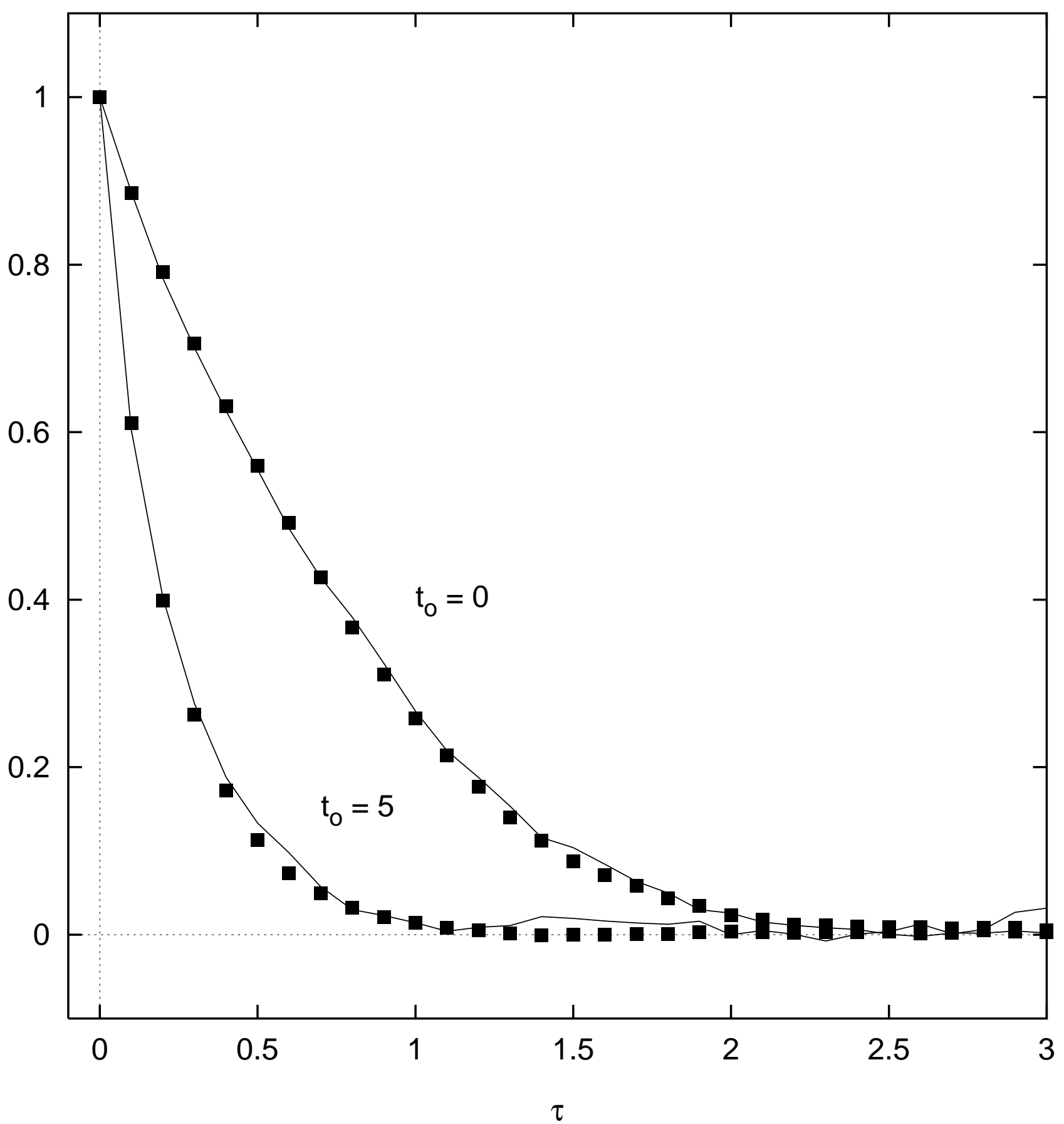

Fig 3 


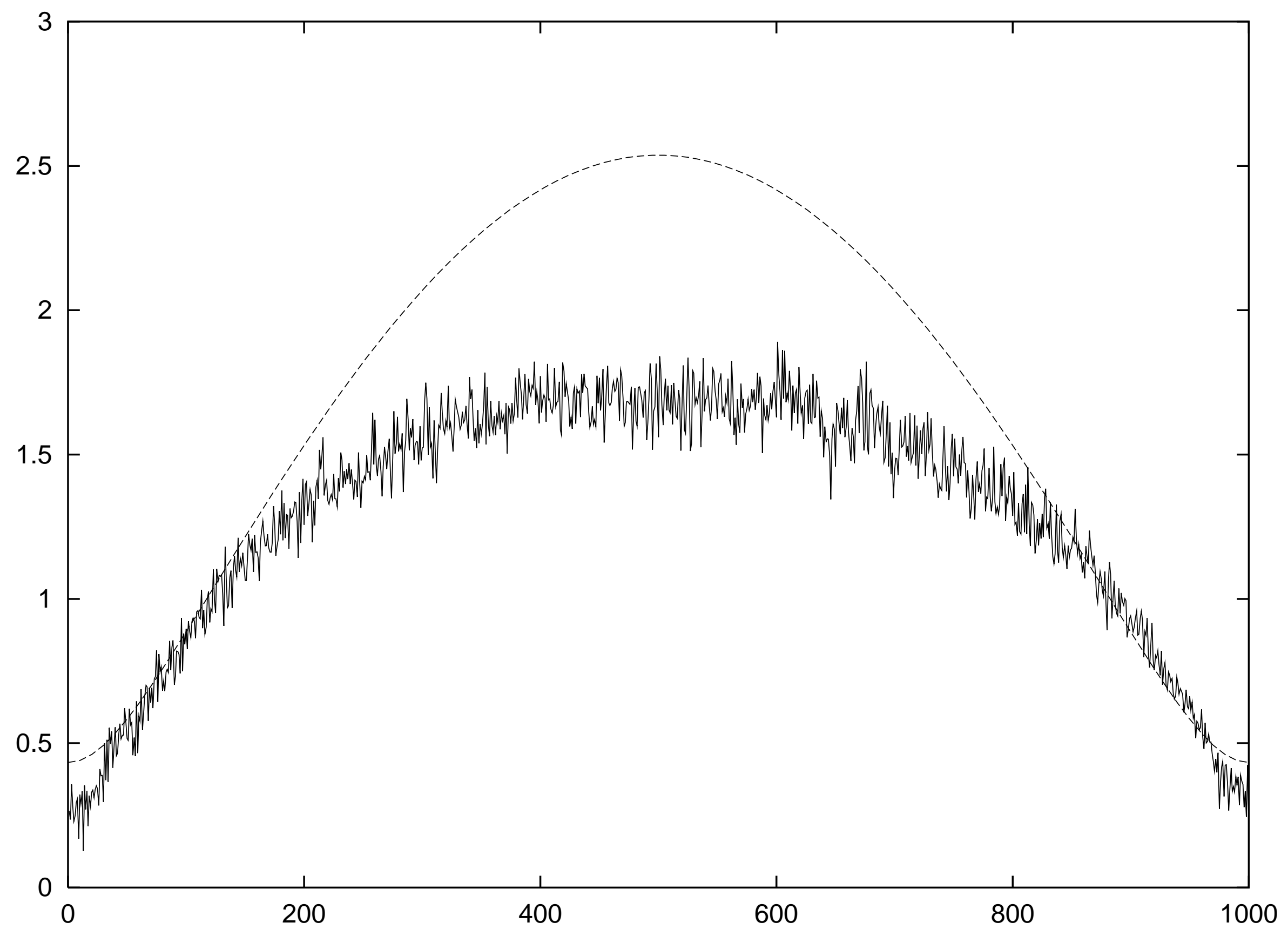




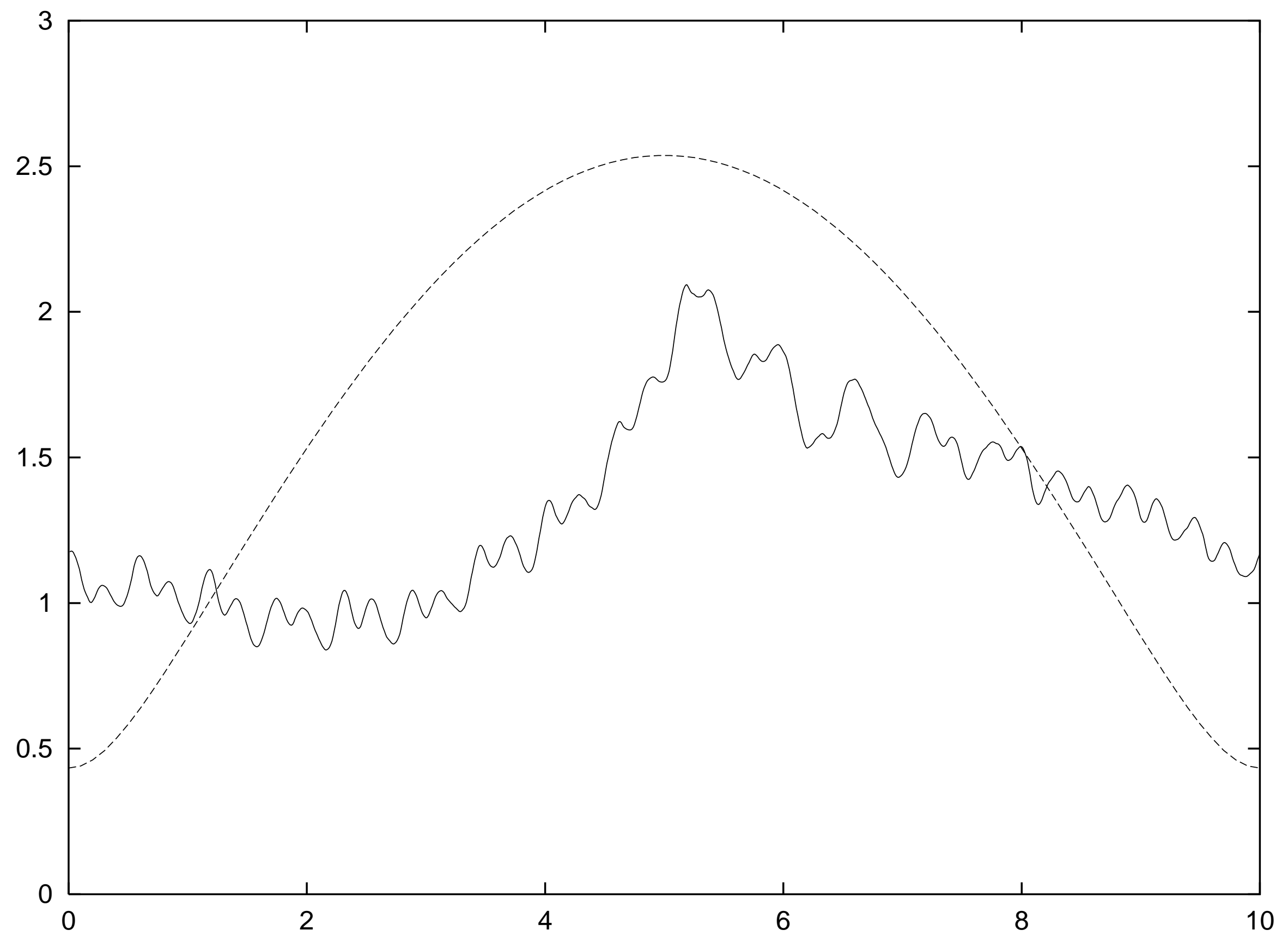




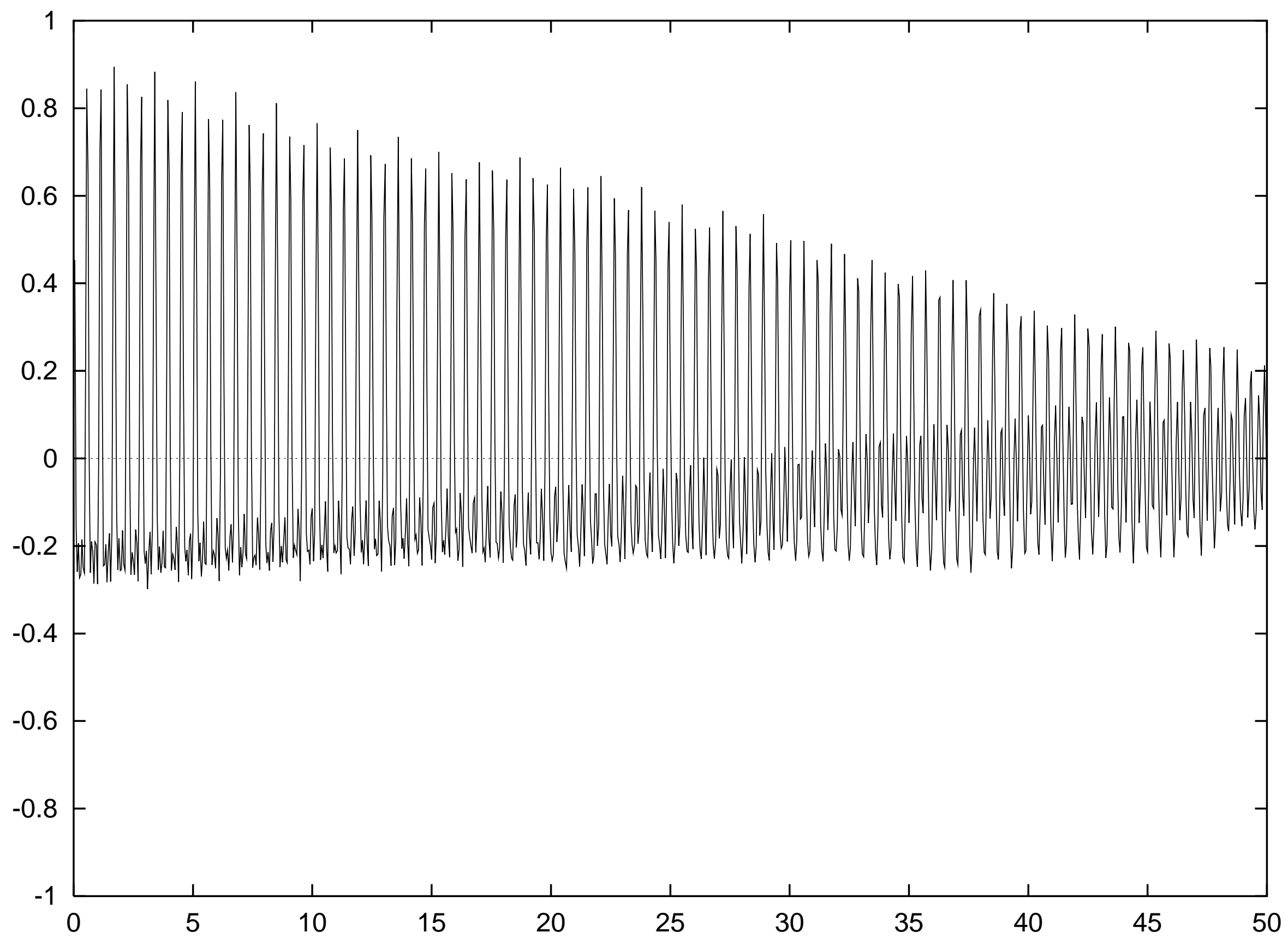




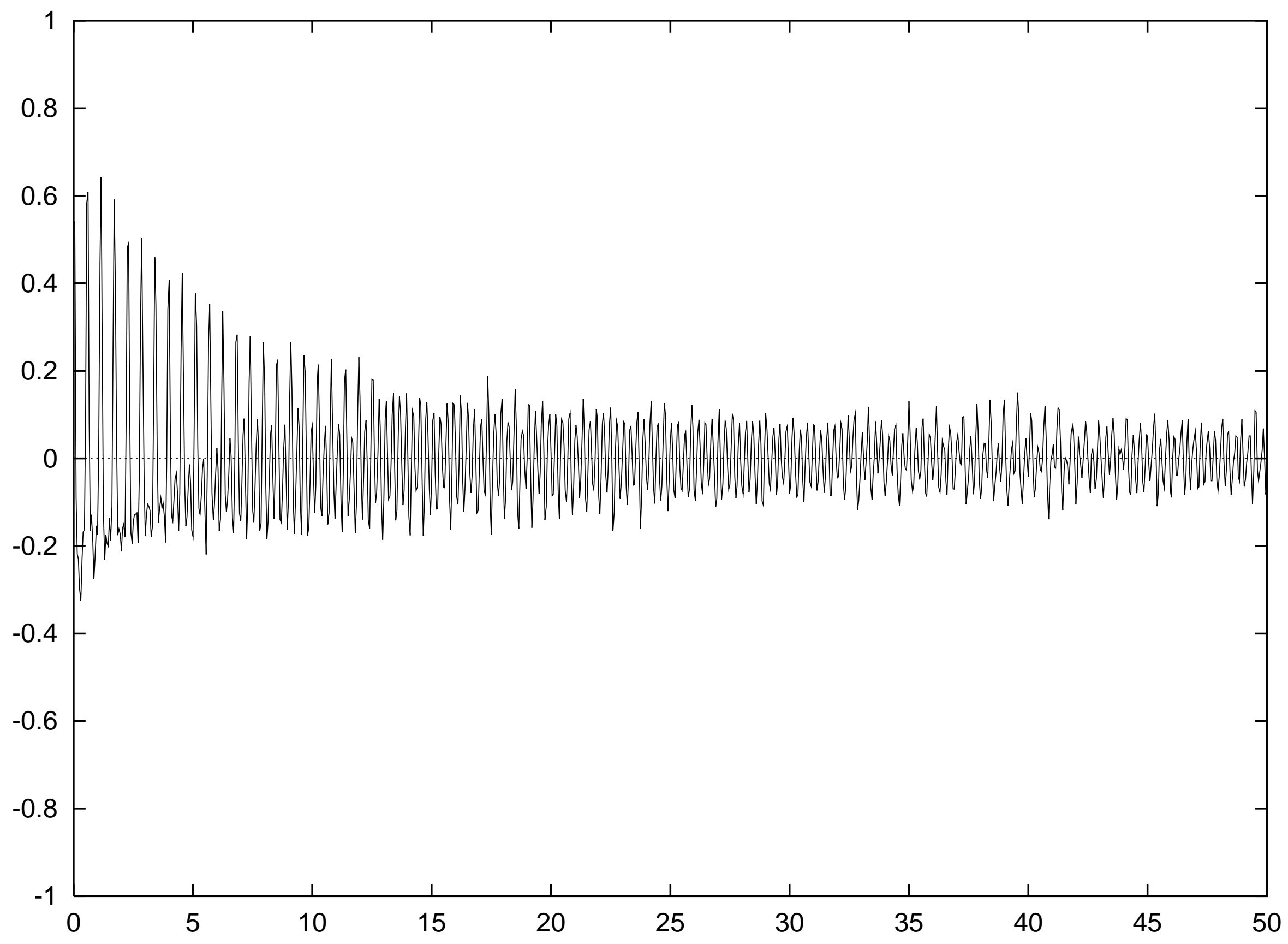

